\title{
VALIDACIÓN DE UNA TECNOLOGÍA APROPIADA PARA EL CULTIVO DE CAPULí (Physalis peruviana L.) PARA LAS CONDICIONES DEL VALLE DEL MANTARO
}

\author{
VALIDATION OF AN APPROPRIATE TECHNOLOGY FOR THE CULTIVATION OF CAPULÍ (Physalis \\ peruviana L.) FOR THE VALLEY THE MANTARO CONDITIONS
}

Doris Marmolejo Gutarra', Edgar Ramírez Valerio', Juan Bullón Ames'

\section{RESUMEN}

Capulí (Physalis peruviana L.) especie nativa del Perú, cultivo restringido en huertos familiares, actualmente viene siendo revalorado por su calidad nutricional y medicinal. Muchas instituciones han tomado importancia por ser un cultivo potencial y alternativo para la población rural, y su gran adaptación a las condiciones ambientales del valle. El trabajo se desarrolló del 2000 al 2003, se consideró como factores en estudio: tipos de sustratos, densidad de siembra, tipos de tutores, usos y manejo agronómico. Los resultados obtenidos es como sigue: el sustrato compost destacó para rendimiento de frutos con 0,422 $\mathrm{kg} /$ planta, para distanciamientos ocupó el primer lugar la densidad 1,20 x 1,00 m con un rendimiento de $0,596 \mathrm{~kg} /$ planta, en el uso de tutores sobresalió la estaca individual con un rendimiento de 1,88 $\mathrm{kg} /$ parcela; según el resultado de la encuesta sobre el uso del capulí el $51 \%$ de la población en la margen izquierda lo destina al autoconsumo y el $37 \%$ como medicina natural; en la margen derecha un $50 \%$ para autoconsumo y el $37 \%$ como medicina natural. Como propuesta de solución al manejo agronómico y usos del capulí, se propone: utilizar una agricultura sostenible. Asimismo, promocionar el consumo en estado fresco y procesado, de esta manera obtener valor agregado.

Palabras clave: Physalis peruviana, tipos, sustratos, manejo agronómico.

\begin{abstract}
Capuli (Physalis peruviana L.) native species of Perú, restricted cultivation in family gardens, at present comes being reevaluated because its medicinal and nutritious quality. Many institutions have taken importance by being an alternative and potential cultivation for the rural population, and their great adaptation to the environmental conditions of the valley. The present study was developed from the 2000 to 2003, it was considered like factors in study: types of substrates, density of sowing, types of tutors, uses and agronomic management. The results obtained were: the substrate compost emphasized for yield of fruits with $0,422 \mathrm{~kg} /$ plant; for distances occupied the first place the density $1,20 \times 1,00 \mathrm{~m}$ with a yield of $0,596 \mathrm{~kg} / \mathrm{plant}$, in the use of tutors the individual stake with a yield of 1,88 $\mathrm{kg} / \mathrm{plot}$, according to the result of the survey on the use of the Capuli the $51 \%$ of the population in the left margin destines to personal use and $37 \%$ as natural medicine; in the right margin a $50 \%$ for personal use and the $37 \%$ like natural medicine. As proposal of solution of agronomic management and uses of Capulí, it is proposed: to use sustainable agriculture. Likewise, to promote the consumption in fresh state and processed, in this manner to obtain aggregate value.
\end{abstract}

Key words: Physalis peruviana, types, substrate, agronomic management. 


\section{INTRODUCCIÓN}

El cultivo del capulí (Physalis peruviana L.) es originario de los Andes del Perú; crece en estado silvestre o como maleza y su cultivo está restringido a huertos caseros, Vallejo, 2000 (4). Su cultivo está limitado a los agricultores de la sierra del Perú de manera aislada, como un cultivo complementario utilizando tecnologías tradicionales, Pacheco, 1999 (3). El capulí es importante en la alimentación por su alto contenido de vitamina $B_{12}$, tiamina, calcio, fósforo, caroteno, ácido ascórbico, y propiedades medicinales, Ugas, 1993 (5) El capulí es consumido en fruto fresco, mermeladas, jugos, néctares, almíbar y helados, Ugas, 1993 (5). Actualmente muchos países vienen cultivando esta especie, Colombia, es el primer productor; Sud África, Nueva Zelanda, India, Hawai y Ecuador. El abonamiento orgánico contribuye en el mejor desarrollo y producción, especialmente en el uso de almacigado, Fisher y Angulo, 1999 (1), el distanciamiento en capulí entre surcos de $1,00 \times 0,80 \mathrm{~m}$ entre plantas reporta buenos rendimientos, Ugas, 1993 (5). Los tutores facilitan las labores agronómicas y cosecha, además permite una mayor entrada de luz, aire en las plantas, favorece guiar a la planta y evitar el encamado de las mismas. Los objetivos fueron: Determinar la tecnología apropiada para el cultivo de capulí para las condiciones del valle del Mantaro; y validar los diferentes componentes de la tecnología en estudio.

\section{MATERIAL Y MÉTODOS}

\section{Factores en Estudio:}

\section{Factor A. Tipos de sustratos:}

Estiércol de vacuno (EV), Compost (C), humus de lombriz $(\mathrm{HL})$ y tierra agrícola

(Testigo).

Factor B. Densidades de siembra:

Distancia entre surcos: $\quad 1,00 \mathrm{~m}\left(\mathrm{~A}_{1}\right), 1,20 \mathrm{~m}$ $\left(A_{2}\right)$, y $1,40 m\left(A_{3}\right)$

Distancia entre plantas: $0,80 \mathrm{~m}\left(\mathrm{~B}_{2}\right)$ y $1,00 \mathrm{~m}\left(\mathrm{~B}_{3}\right)$.

$0,60 \mathrm{~m}\left(\mathrm{~B}_{1}\right)$,

Factor C. Tipos de tutores

En espaldera, estacas individuales y sin tutor.

Factor D. Incidencia de plagas $y$ enfermedades:

Evaluación en las zonas agroecológicas bajas e intermedia según Mayer (1981).

Factor E. Usos y manejo agronómico

Mediante encuestas directas en las zonas se estudio.

\section{Diseño Experimental}

Bloque completamente al azar, con 4 repeticiones.

\section{Conducción de Experimento}

Tipos de sustratos: $50 \%$ de estiércol de vacuno más $50 \%$ de tierra agrícola; $50 \%$ de compost más $50 \%$ de tierra agrícola; $50 \%$ de humus de lombriz más $50 \%$ de tierra agrícola y como testigo tierra agrícola al $100 \%$.

\section{Densidades de siembra:}

Distancia entre surcos:

$\left(A_{2}\right)$, y $1,40 m\left(A_{3}\right)$

Distancia entre plantas:

$\left(B_{2}\right)$ y $1,00 \mathrm{~m}\left(B_{3}\right)$

Tipos de tutores. En espaldera, estaca individual y sin tutor (testigo). Los tutores se instalaron cuando las plantas alcanzaron $30 \mathrm{~cm}$. de altura.

Labores culturales. Durante el almacigado se realizaron deshierbos y riegos de acuerdo a la exigencia del cultivo. Después del trasplante a campo definitivo se efectuaron los riegos, deshierbo y el aporque.

Cosecha. De manera gradual según la maduración de los frutos en los diferentes tratamientos en estudio.

Encuestas de usos y manejo agronómico. Se realizaron encuestas directas a los agricultores de las zonas en estudio.

\section{RESULTADOS Y DISCUSIÓN}

En la tabla 1, de la prueba de significación de los promedios del peso de fruto en $\mathrm{kg} /$ planta en tipos

\begin{tabular}{|c|c|c|c|c|c|}
\hline o.h. & Silstratos & Clave & $N^{c}$ Trat. & Kajplta. & Significaciōn \\
\hline ; & $\begin{array}{l}\text { r-cmpost } \\
\text {-Lmus de }\end{array}$ & r: & 2 & $0,4 \%$ & A \\
\hline 2 & $\begin{array}{l}\text { lomorlz } \\
\text { t=tiéccl de }\end{array}$ & $\mathrm{HL}$ & 3 & 0,343 & $\forall$ \\
\hline 3 & vac Jno & $E$ & 1 & 0,33 & $\forall$ \\
\hline 4 & Tierra agríc sa & Toot go & 4 & 0,266 & c \\
\hline
\end{tabular}


de sustratos, se observa que el tratamiento Compost más tierra agrícola ocupa el primer lugar según orden de mérito, con un promedio de $0,422 \mathrm{~kg} /$ planta, debido al efecto de mejor disponibilidad de nutrientes a la planta repercutiendo en el vigor, consecuentemente mejor rendimiento.

En la tabla 2, de la prueba de significación de los promedios de rendimiento de frutos en densidades de siembra, se observa que, para la interacción de los distanciamientos entre surcos y plantas los tratamientos A2B3, A2B1 y A2B2 ocupan los tres primeros lugares según el orden de mérito con 0,596; 0,591 y $0,589 \mathrm{~kg} / \mathrm{planta}$ respectivamente, debido a que los distanciamientos entre surcos y plantas permitieron un mayor desarrollo por efecto de mejor aprovechamiento de la iluminación solar, nutrientes, agua y espacio.

\section{ractor $B$ : Uensidades de Siembra}

Tabla 2. Prueka de significación de los promedias del rendimiento te frutos de captl (kyjlanta) para tencirlart re sipmbra, sfglin TI kFy

\begin{tabular}{|c|c|c|c|c|}
\hline O.M. & Densidades im & $N^{\circ}$ Trat. & Rend. Kgipla. & Signnificación \\
\hline 1 & A.2日3 $(1,20 \times 1, C 0)$ & 6 & 0,596 & A \\
\hline 2 & A.2B। $(1,20 \times 0, E 0)$ & $\angle$ & 0,591 & A. \\
\hline 3 & A2B2 $(1,20 \times 0,80)$ & 5 & 0.589 & A. \\
\hline 4 & A 3 B $3(1,40 \times 1, C 0)$ & 9 & $0,4 \overline{0} 0$ & $\mathrm{~B}$ \\
\hline 5 & $A^{\prime} B 2(1,00 \times 0, \varepsilon 0)$ & 2 & 0,455 & $\mathrm{~B}$ \\
\hline 6 & A3B1 $(1,10 ; \times(0,60)$ & T & $0,1.19$ & B \\
\hline 7 & $\mathrm{~A} 3 \mathrm{~B} 2(1,40 \times 0,80)$ & 8 & 0,386 & c \\
\hline 8 & A. B1 $(1,00 \times 0, E 0)$ & 1 & 0,381 & c \\
\hline 9 & N. $\mathrm{B} 3(1,00)(1, \mathrm{C})$ & 3 & $0,3^{75}$ & $c$ \\
\hline
\end{tabular}

\section{Factor D: Evaluación de Plagas y Enfermedades}

En la zona de estudio se detectó las siguientes plagas: Epitrix sp., en las localidades de: Pilcomayo, Mantaro, Concepción, Ataura, Apata y Matahuasi; Diabróticas spp., en las localidades de Ataura, Pilcomayo, Chupaca, Sincos y Mantaro; Myzus sp., en las localidades de Mantaro, Concepción, Chupaca, Vilcacoto, Pilcomayo y San Jerónimo; Liriomyza huidobrensis, Dicyphus curcubitaceus, en las localidades de Mantaro, Huertas, Masma, Ataura, Jauja y Concepción. Pilcomayo, Sincos, Matahuasi, Mantaro, Ataura y Concepción. Las patógenos detectadas en las zonas de estudio fueron: Botrytis sp., en las localidades de Concepción, Apata, Mantaro, Matahuasi y Concepción; Fusarium sp., en las localidades de Mantaro, Ataura, Concepción, Matahuasi, Sincos y Pilcomayo.

\section{Factor E: Usos y Manejo Agronómico}

De acuerdo al resultado de la encuesta realizada en las zonas en estudio Jauja, Concepción,

En la tabla 3, de la prueba de significación de los promedios del peso de frutos en $\mathrm{kg} /$ planta para tipos de tutores, se observa que, el tratamiento T2 (estaca individual) ocupa el primer lugar según orden de mérito con 1,88 kg/parcela; seguido del tratamiento T1 (espaldera) con 1,37 kg/parcela; debido al mejor crecimiento del tallo y las ramas favorecido por el sostenimiento de los tutores, por el crecimiento decumbente de las plantas, de esta manera permite la buena fructificación y calidad de los frutos.
Matahuasi, El Mantaro, Ataura, San Jerónimo, Pilcomayo, Chupaca, Chongos Bajo, Huertas, Masma, Apata, Ingenio, Vilcacoto), para el uso del fruto del capulí el $51 \%$ de la población en la margen izquierda lo destina al autoconsumo y un $37 \%$ como medicina natural; en la margen derecha reporta un $50 \%$ de la población lo destina para autoconsumo y un $37 \%$ como medicina natural respectivamente. Para la venta en mercados locales en la margen izquierda es del 9\% y en la margen derecha del $10 \%$. Para procesamiento es del 3\% tanto para la margen izquierda y derecha. 
Tabla 4. Problemas sobre el Manejo Agronómico y Usos del capulí (Physalis

MAHEJO AGRONÓMICO DEL Physalis penuviana $L$.

$\checkmark$ Planta considerada comc maleza, por el cual no se le da ningún tipo de atenciór en su cultivo.

$\checkmark$ Desconocimiento de la fenología, śpoca de sie nbre, menejc de almácigo y conducción en campc definitivo.

$\checkmark$ Germinación desuniforme de las semilles por la variabilidad genétiza existentes

$\checkmark$ Desconocimiento de densidades de siembres adecuadas.

$v^{\prime}$ Desconocimiento de dosis óptima ce abonamiento.

$\checkmark$ Hábito de crecimento decumbente.

$\checkmark$ Desconocimiento de podas de formación.

\Dańos ocasionacos por plagas y enfermedades potencialas.

$\checkmark$ Susceptbilidad a las heladas en la fbrac ón y fructificación, ocasicnando ladídid de flures y frulus.

\section{USOS}

$\checkmark$ Docconocimionto del valor nutricioral del fruto del casuli.

$\checkmark$ Falta de información sobre el consumo en frescc y procesado del producto.

$\checkmark$ Lesconocimiento del manejo de cosecha, post cosachay conservación de los rutos. penuviana L.) en las zonas en estudio, según encuesta.

$\checkmark$ Obtener semillas de frutos seleccionados por uniformidad en tamaño, color y libre de enfermedades.

$\checkmark$ Utilizar sistemas de cultivo asociado, intercalado con cultivos de la zona, para el mejor uso efectivo del suelo.

$\checkmark$ Evitar competencias con malezas anuales.

$\checkmark$ Usar abono orgánico con la finalidad de producir frutos ecológicos.

$\checkmark$ Para el control de aves utilizar c intas $\mathrm{m}$ a g n e tofón i c a s distribuidas en todo el campo, también las técnicas de ultrasonidos utilizados en el Ecuador.

$\checkmark$ Realizar la cosecha de

Alternativas de solución para el manejo agronómico y usos del Capulí (Physalis peruviana L.).

\section{Manejo Agronómico: Alternativas de solución:}

$\checkmark$ Realizar evaluaciones del comportamiento en almácigo, trasplante, en campo definitivo, en la cosecha y manejo post cosecha.

$\checkmark$ Usar cama de almácigo adecuado utilizando materia orgánica como sustrato.

$\checkmark$ Utilizar cobertores en los almácigos en épocas de heladas.

$\checkmark$ Realizar el trasplante en época oportuna y de preferencia por las mañanas.

$\checkmark$ Preparar adecuadamente el terreno de cultivo de manera anticipada.

$\checkmark$ En campo definitivo adicionar principalmente fuentes de materia orgánica.

$\checkmark$ Realizar aporques altos para evitar el contacto de los frutos con el suelo, evitando la pudrición.

$\checkmark$ Efectuar los riegos de acuerdo a los requerimientos del cultivo.

$\checkmark$ Utilizar tutores para la mejor aireación, captación de los rayos solares y evitar presencia de enfermedades, de esta manera obtener buenos rendimientos y calidad de frutos.

$\checkmark$ Realizar podas de formación con la finalidad de lograr un balance de crecimiento y producción de frutos. manera oportuna, utilizando tijeras en la recolección de frutos depositando en cestos.

$\checkmark$ Los frutos cosechados llevar a la sombra sobre yutes o mantadas para la clasificación correspondiente y luego empacar en cajas especiales para ser transportado a los mercados locales y nacionales.

$\checkmark$ Inmediatamente después de la cosecha es conveniente el tendido de los brutos, con la finalidad de que el cáliz pierda humedad, de esta manera evitar la pudrición.

\section{Usos: Alternativas de solución:}

$\checkmark$ Promocionar el consumo en las zonas urbanas rurales, especialmente en los niños y adulto mayor.

$\checkmark$ Consumir en fruto fresco, mermeladas, jugos, néctares, almíbar, helados.

$\checkmark$ Promocionar y realizar degustaciones, para dar a conocer las propiedades nutricionales y medicinales.

\section{CONCLUSIONES}

1. El sustrato compost destacó para el peso de fruto con $0,422 \mathrm{~kg} /$ planta, debido al efecto de mejor disponibilidad de nutrientes, de esta manera influyendo en el mejor vigor y en consecuencia mayor rendimiento.

2. Los distanciamientos $1,20 \times 1,00 ; 1,20 \times 0,60$ y $1,20 \times 0,80 \mathrm{~m}$ ocuparon los tres primeros 
lugares con un rendimiento de 0,$596 ; 0,591$ y $0,589 \mathrm{~kg} /$ planta respectivamente, debido al mejor aprovechamiento de la iluminación, nutrientes, agua y espacio.

3. El uso de tutor estaca individual ocupó el primer lugar con un rendimiento de $1,88 \mathrm{~kg} /$ parcela, seguido del tratamiento en espaldera con 1,37 $\mathrm{kg}$ /parcela., debido al mejor crecimiento del tallo y ramas favorecido por los tutores.

4. En la zona agroecológica en estudio se registraron las siguientes plagas Diabrótica spp, Liriomyza huidobrensis; Epitrix sp, Myzus sp y Dicyphus cucurbitaceus. Los patógenos registrados fueron: Botrytis sp y Fusarium sp.

5. Según el resultado de la encuesta sobre el uso del fruto del capulí el $51 \%$ de la población de la margen izquierda lo destina al autoconsumo y un $37 \%$ como medicina natural; en la margen derecha reporta un $50 \%$ para autoconsumo y un $37 \%$ como medicina natural respectivamente.

6. La encuesta reporta que el manejo agronómico presenta muchas deficiencias, como el uso inadecuado de almacigado, desconocimiento de densidades, podas, tutores, abonamiento, control de plagas y enfermedades, cosecha y post cosecha,
7. Como propuesta de solución al manejo agronómico y usos del capulí, se propone: utilizar una agricultura sostenido de acuerdo a las condiciones agroclimáticas del valle del Mantaro. Asimismo, promocionar el consumo en estado fresco y procesado, de esta manera lograr el valor agregado.

\section{REFERENCIAS BIBLIOGRÁFICAS}

1. Fischer, Angulo. Los frutales de clima frió en Colombia: la uchuva. Ventana al campo andino. Colombia; 1999.

2. Liñan, J. Los frutales nativos del Perú. Riqueza desaprovechada.Lima: Editorial UNMSM. EEA La Molina; 1996.

3. Pacheco A; J. Saavedra O. Awaymanto, Physalis peruviana L. Misión Rural. Lima: CARESPERU.15-19 p; 1999.

4. Vallejo C. Producción comercial de uvilla. Ecuador; 2000.

5. Ugas R, Antonio J, J Barrenechea, M Sevilla. El capulí. Revista Agronomía. Lima: Volumen XLI. 1993: 25 -30.

Email: Dorismg33@hotmail.com 